ERRATUM

\title{
Validation of the Cohen-Mansfield Agitation Inventory Observational (CMAI-0) tool - ERRATUM
}

\author{
Alys W. Griffiths, Christopher P. Albertyn, Natasha L. Burnley, Byron Creese, \\ Rebecca Walwyn, Ivana Holloway, Jana Safarikova, and Claire A. Surr
}

Key words: dementia, agitation, observation, care homes, proxy bias; erratum

DOI: https://doi.org/10.1017/S1041610219000279, Published by Cambridge University Press, 10 April 2019

The above article (Griffiths et al., 2019) published with an incorrect abstract.

The correct abstract is as follows:

Objectives: Behaviours associated with agitation are common in people living with dementia. The CohenMansfield Agitation Inventory (CMAI) is a 29-item scale widely used to assess agitation completed by a proxy (family carer or staff member). However, proxy informants introduce possible reporting bias when blinding to the treatment arm is not possible, and potential accuracy issues due to irregular contact between the proxy and the person with dementia over the reporting period. An observational measure completed by a blinded researcher may address these issues, but no agitation measures with comparable items exist.

Design: Development and validation of an observational version of the CMAI (CMAI-O), to assess its validity as an alternative or complementary measure of agitation.

Setting: Fifty care homes in England.

Participants: Residents $(N=726)$ with dementia.

Measurements: Two observational measures (CMAI-O and PAS) were completed by an independent researcher. Measures of agitation, functional status, and neuropsychiatric symptoms were completed with staff proxies.

Results: The CMAI-O showed adequate internal consistency $(\alpha=.61)$, criterion validity with the PAS $(r=.79$, $p=<.001)$, incremental validity in predicting quality of life beyond the Functional Assessment Staging of Alzheimer's disease $(\beta=1.83, p<.001$ at baseline) and discriminant validity from the Neuropsychiatric Inventory Apathy subscale $(r=.004, p=.902)$.

Conclusions: The CMAI-O is a promising research tool for independently measuring agitation in people with dementia in care homes. Its use alongside the CMAI could provide a more robust understanding of agitation amongst residents with dementia.

The original article has been corrected online to rectify this error.

\section{Reference}

Griffiths, A. W., Albertyn, C. P., Burnley, N. L., Creese, B., Walwyn, R., Holloway, I., Safarikova, J. and Surr, C. A. (2019). Validation of the Cohen-Mansfield Agitation Inventory Observational (CMAI-O) tool. International Psychogeriatrics, doi: 10.1017/S1041610219000279. 